\title{
Melisa MĂRCUȘ
}

\section{La phénoménologie de la perception mélancolique. La mélancolie sentie dans le cinéma-corps}

\begin{abstract}
This paper discusses melancholy as an existential solitude that is acknowledged and amplified through the cinematic experience. In order to prove that this feeling is not only a psychological distress, but it is mainly perceived through the manifestations of the body and, paradoxically, through interaction rather that isolation, we will rely on the strong connection between phenomenology and the cinematic perception, as highlighted by film theorists and by philosopher Maurice Merleau-Ponty, but also on some of the ideas belonging to existential philosopher Jean-Paul Sartre that we have thought suitable for the analysis of two melancholy-toned films: Melancholia (2011), directed by Lars von Trier and Solaris (1972), by Andrei Tarkovsky.
\end{abstract}

Keywords: cinema; body; perception; Melancholia; Solaris; melancholy; phenomenology; reflections; solitude; absence.

Observer la mélancolie dans un produit artistique pourrait paraître un geste superflu, car elle a souvent constitué le thème ou le ton d'un œuvre d'art et c'est pourquoi il faut commencer par souligner en quoi le cinéma se différencie des autres formes d'expression artistique dans l'évocation de ce sentiment et pourquoi elle mérite encore d'occuper la place centrale de notre recherche. Bien qu'elle ait été exprimée en mots, en couleurs, en sons, ou seulement ressentie dans la réception d'un œuvre, elle n'a jamais été vivante dans l'art. En cela réside le pouvoir du cinéma : il crée l'impression que la mélancolie est réellement présente et vécue au moment de sa perception.

Melisa MĂRCUȘ

Babeș-Bolyai University

melisa_marcus@yahoo.com

EKPHRASIS, 1/2019

A Melancholic Exploration of Humanity

(The Solitude of Man)

pp. 59-69

DOI:10.24193/ekphrasis.21.5

Published First Online: 2019/06/27 
Il crée l'illusion de la réalité par le mouvement des formes et des images. Dans son essai qui approche les films d'une manière phénoménologique, intitulé « À propos de l'impression de réalité au cinéma », le théoricien Christian Metz explique le sentiment d'un " être-là vivant » et d'une corporalité des formes que le mouvement crée dans l'art cinématographique, à la différence d'autres arts.

Le choix d'examiner la mélancolie à travers les manifestations externes d'un corps au lieu de considérer les troubles internes qu'elle implique est ce qui nous conduit vers une approche phénoménologique et existentielle de sa perception. Maurice Merleau-Ponty, dans sa conférence « Le cinéma et la nouvelle psychologie », distingue la psychologie classique, qui décrit un sentiment par l'introspection, de la psychologie moderne, qui s'appuie sur la conduite visible et sur les manifestations externes des émotions pour comprendre les sentiments. Le philosophe soutient que la dernière convient mieux au cinéma, car il peut s'en servir pour rendre visible les sentiments dans leur forme concrète : "Colère, honte, haine, amour ne sont pas des faits psychiques cachés au plus profond de la conscience d'autrui, ce sont des types de comportement ou des styles de conduite visibles du dehors. Ils sont sur ce visage ou dans ces gestes et non pas cachés derrière eux » (Merleau-Ponty 66). La même idée se trouve dans l'essai d'ontologie phénoménologique L'être et le néant de Jean-Paul Sartre, où le psychisme ne peut exister ou se manifester sans une structure corporelle avec laquelle il se confond :

[...] ces manifestations émotionnelles [...] ne nous indiquent nullement une affection cachée et vécue par quelque psychisme, qui serait l'objet immatériel des recherches du psychologue : ces froncements de sourcils, cette rougeur, ce bégaiement, ce léger tremblement des mains [...] n'expriment pas la colère, ils sont la colère. (388)

En considérant la mélancolie comme une de ces manifestations qui peuvent prendre charge d'un corps pour se rendre visibles, on se propose d'analyser les films comme des corps dont chaque mouvement et chaque «tremblement » s'identifient avec l'état psychique. On arrive ainsi à encore un des traits spécifiques du cinéma par rapport à la littérature. Si la littérature décrit plutôt la vie intérieure des personnages, les films peuvent illustrer la philosophie de Sartre, où la conscience est inséparable du corps : c'est par le corps et par la relation avec le monde et avec les autres que l'homme arrive à se connaître. Il ne peut pas se définir lui-même que s'il apparaît à autrui pour qu'il le reconnaisse. Cette relation de définition réciproque et les troubles qu'elle engendre est fortement visible dans Solaris, plus précisément dans le couple Hari-Kris Kelvin. Le film de Tarkovski arrive à exprimer la mélancolie en l'incarnant dans les corps des personnages et dans le cinéma-corps, dans un style caractéristique au réalisateur considéré comme « le poète du cinéma ».

Solaris fait sentir aux personnages et à nous une solitude associée à la séparation et à l'absence. S'il n'est pas le seul film d'Andreï Tarkovski imprégné d'une nuance mélancolique, il se remarque toutefois par l'ampleur du sentiment. La mélancolie est 
intensifiée, d'un côté, par le dépassement des bords de la Terre - le film nous présente un voyage dans le Cosmos qui a comme but l'étude de la planète Solaris et, de règle, ces voyages sont connus comme de grands déclencheurs de troubles psychiques. De l'autre côté, la mélancolie peut être vue comme un sentiment inhérent au cinéphile, dans une lecture censée déchiffrer la métaphore de l'expérience cinématographique que le film déploie. À un niveau de base, on peut observer la séparation et l'absence dans la structure et la trame du film. Dans la première partie, qui se déroule sur la Terre, Kelvin ressent l'absence de sa mère et de son épouse décédées et la séparation de son père. En même temps qu'il semble vouloir garder la séparation de son passée (il brûle des papiers et des photos anciennes, il est distant par rapport à son père), Kelvin veut aussi combler l'absence ressentie et il décide de s'embarquer pour une mission dans l'espace. Cependant, il s'avère qu'il ne réussit pas à dépasser sa solitude même après que la planète Solaris lui a permis de revoir sa femme. Une possible explication pour ce résultat se trouve à un niveau d'interprétation méta-cinématographique, en attribuant à Solaris une auto-référentialité tout à fait originelle, non-intentionnelle, qui porte non seulement sur la réalisation d'un film, mais surtout sur les mécanismes de perception cinématographique.

Dans son livre, The Solaris Effect: Art and Artifice in Contemporary American Film, Steven Dillon traite le sujet du dévoilement de l'illusion propre au cinéma, que Solaris exemplifie à travers la relation entre Hari et Kris. Dans ce couple, Hari est pris pour l'image présente et absente à la fois que l'écran projette dans la salle de cinéma, tandis que Kris s'identifie au spectateur, mais aussi au créateur du film. La raison pour laquelle Kris persiste dans sa mélancolie tient du fait qu'il sait à chaque moment que Hari n'est pas réelle, qu'elle est seulement une reproduction mécanique (fig. 1), comme le cinéma même, où on perçoit comme étant présentes des réalités qui ne sont plus là :

Kris's love for Hari is like our impossible desire for cinema. What we desire is contact and communication, but both the monitors and Hari

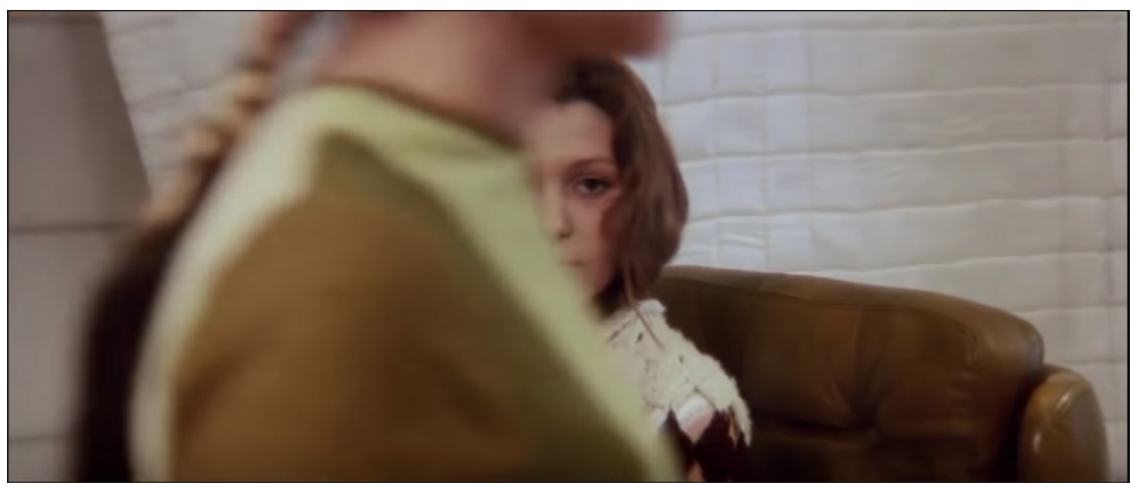

Fig. 1. Capture d'écran de Solaris, où Hari apparaît comme une reproduction mécanique. Glissant autour de la chambre, la caméra la perçoit dans plusieurs endroits. 
ironically emphasize our solitude. We would recognize our solitude less, perhaps, if we were simply alone. (Dillon 9)

Ainsi, la solitude est ressentie aussi par le cinéphile au moment de la perception du film, toujours par la séparation, dans ce qu'Albert Michotte nomme «la ségrégation des espaces » (apud. Metz 1975 : 20), c'est-à-dire la distance entre la réalité de la salle de cinéma et l'univers diégétique du film, dont le spectateur est toujours conscient, et par l'absence - comme dans le cas de Hari, les personnages du film ont une présence fantomatique et c'est le spectateur qui peut les investir d'une réalité qui vient de ses projections. La nouvelle Hari est juste une copie de la femme de Kris, elle est nommée « reproduction », " matrice » par les scientifiques et tous admettent, elle aussi, que la vraie Hari est morte. De cette façon, elle est semblable à un film dont les images vivent chaque fois qu' on les projette et dont l'existence dépend de nous, tout comme l'image de Hari dépend de Kris.

De même, le couple Hari-Kris traduit, toujours par l'incarnation, le jeu de reflets qui se trouve dans le processus des perceptions ordinaires et cinématographiques, mais aussi dans la relation entre l'aimant et l'aimé et dans la construction du sujet, tel que décrites par Jean-Paul Sartre, qui nous permettront de voir la solitude existentielle sous forme d'aliénation de soi-même. Dans Le signifiant imaginaire. Psychanalyse et cinéma, Christian Metz décrit la construction d'une vision du monde par un double mouvement de projection et d'introjection :

J'ai à la fois l'impression de « jeter », comme on dit, mon regard sur les choses, et que ces dernières, ainsi illuminées, viennent se déposer en moi (on déclare alors que ce sont elles qui se "projettent » : sur ma rétine par exemple). Il faut répandre sur le monde une sorte de flot, qui s'appelle le regard [...] pour que les objets puissent remonter ce flot en sens inverse (71).

Ce processus imite la perception du film dans la salle de cinéma, aussi que la réalisation du film. Hari peut être pris pour l'écran du cinéma qui montre ce que le directeur a enregistré, c'est-à-dire ce que Kris se rappelle à propos d'elle. On trouve ici un autre type de séparation et d'absence : le percevant et le perçu ne se rencontrent jamais ; quand le spectateur perçoit l'image, elle n'est pas réellement là, elle est reçue comme enregistrée dans un autre lieu et temps, ou, selon André Bazin, comme «la présence-absence du représenté » (apud. Dillon 2).

En ce qui concerne la solitude comme séparation et aliénation inhérentes à la construction du moi et à la relation d'amour du point de vue phénoménologique, on la retrouve dans l'essai de Hari de se connaître à l'aide de Kris. Sartre affirme que la certitude d'une conscience de soi n'est pas vraie dans l'absence d'un autre qui la reflèterait à la manière d'un écho (276). Pourtant, entre les deux consciences, il y a toujours un néant, une distance nécessaire pour le fondement de leur relation : «l'autre est ce qui m'exclut en étant soi, ce que j'exclus pour être moi » (275). Hari ne peut pas se définir en se regardant dans le miroir, elle a besoin de Kris pour la reconnaître. 
Dans une lecture lacanienne, le critique Christian Metz compare l'expérience de Hari à une deuxième étape du stade du miroir : pareil à l'enfant, elle s'est déjà constituée dans le miroir comme objet, elle est maintenant là, elle est l'écran dans le cinéma, mais elle est seulement une illusion. C'est la tâche du spectateur de la définir en totalité, de construire ce que Metz appelle le «signifiant du cinéma » ${ }^{4}$. Cependant, il ne construit pas l'image dans l'écran-miroir comme objet, mais comme sujet, et ainsi il s'identifie plutôt à la caméra. L'incertitude concernant la source de la projection persiste tout au long du film : est-ce que c'est l'Océan de Solaris qui perturbe la perception des chercheurs ou est-ce à cause des encéphalogrammes de ceux-ci qu'apparaissent les hallucinations?

Pour Sartre, la relation d'interdépendance et de construction réciproque entre le moi et l'autre ne suffit pas, car pour lui, le jeu de réflexions va plus loin. Ce jeu d'échos est visible dans le couple de Solaris. Chose notable chez Hari, elle demande Kris très souvent s'il l'aime, s'il avait aimé d'autres femmes dans son absence, elle ne veut pas que Kris la voie comme dégoûtante et elle attribue son caractère humain à la possession de sentiments. On pourrait motiver son attitude en prenant en considération la vision sartrienne sur l'amour - un projet de récupération de son être qui implique l'assimilation de la liberté de l'autre, en tant que liberté aliénée de manière volontaire. Vouloir être aimé, c'est se laisser recréer par autrui comme regard, c'est-à-dire en tant qu'il garde son altérité. L'amour pourrait aboutir à la justification de l'existence et au fondement de la facticité de l'être, mais Sartre fait la preuve de l'impossibilité de l'amour à cause du jeu de reflets à l'infini qu'il implique ${ }^{5}$ et ainsi Hari demeure aussi dans un état mélancolique résultant de l'aliénation au monde et à soi-même.

Comme symbole pour tout ce qu'on a traité à propos de Solaris, il y a le miroir, qui fait preuve de l'illusion qu'implique le cinéma et qui peut être pris pour l'instrument fondamental de la perception cinématographique. Il est mentionné explicitement dans Solaris par Snaut, qui dit: « Nous ne voulons absolument pas conquérir un quelconque Cosmos, mais repousser les frontières de la Terre jusqu'à ses confins. Nous ne savons que faire des autres mondes. Nous n'en avons pas besoin. Nous avons besoin d'un miroir ", réponse qu'on peut interpréter comme suggérant, en rapport avec le sujet du film, les limites de la connaissance humaine. Dans une perspective plus générale, elle peut impliquer les limitations que le cinéma et chaque art doit s'imposer pour qu'ils gardent leurs traits spécifiques, sans risquer de se transformer dans une autre chose. Même si le cinéma a parfois l'air d'englober d'autres arts mis en mouvement et on peut y trouver aussi de différents produits artistiques, on reconnait toujours quand il s'agit d'un film et non d'un enregistrement d'une pièce de théâtre où d'une documentation de tableaux sur fond musical.

Dans un autre sens, la limite est mise en question par le réalisateur Lars von Trier à travers le second film qu'on a choisi d'analyser, Melancholia. Von Trier est connu comme un des fondateurs du Dogme95, un mouvement qui soutient la nécessité 
d'imposer des limites dans la réalisation du film. Dans ce qui suit, nous allons montrer comment von Trier voit l'art cinématographique, en nous appuyant sur le dialogue du film avec certains œuvres d'art et en même temps, nous allons discuter la mélancolie en rapport avec son style et avec la perception cinématographique dans l'acception de Maurice Merleau-Ponty.

Les limites que les cinéastes du mouvement de l'année 1995 s'imposent dans la réalisation du film se réfèrent aux décors simples, à l'équipement réduit, à la lumière non-artificielle et à l'absence de "dollies », en favorisant l'improvisation et la caméra qui dépend seulement des mains des réalisateurs. Cela a comme résultat un film qui donne l'impression de réalité et qui ressemble à un documentaire dont on reconnaît le caractère fictionnel. Selon Gérard Betton, l'ambiguïté du réel est ce qui caractériserait un cinéma de type phénoménologique ou réaliste existentialiste, capable de diminuer la distance entre un cinéma réaliste vraisemblable et un cinéma idéaliste fantastique (Betton 14). En fait, la phénoménologie comme philosophie se trouve aussi à la rencontre de l'idéalisme et de l'empirisme. Plus évident dans d'autres films de Lars von Trier, le mélange réalité-fiction se retrouve aussi dans Melancholia, plutôt sous la forme des contrastes sur les plans technique et esthétique et dans l'illustration de la mélancolie.

Un premier contraste se trouve au niveau des mouvements de la caméra et des points de vue qu'elle prend. On observe que dans les épisodes qui se déroulent à la fête de noces, la caméra est utilisée dans un style qui rappelle le Dogme95 : tenue à la main, elle semble se déplacer parmi les invités, comme si elle prenait part à la fête, ce qui produit une image tremblante, parfois mal cadrée, tandis que dans le reste du film la caméra semble très bien contrôlée et les images comportent des effets spéciaux qui font penser à un film de science-fiction, surtout à cause de la présence de la planète Melancholia et des effets dévastateurs qu'elle anticipe. Ce contraste nous sert d'exemple pour la différence entre la perception ordinaire et la perception cinématographique, une autre observation de Maurice Merleau-Ponty, soulignée par Célia Zernik dans son article. La perception cinématographique a ceci de particulier qu'elle ne présente pas des «bavures », elle est la version idéalisée de la réalité :

Ce bougé, qui exprime le trouble du contact du moi et du monde, est absent de la perception cinématographique. Si la forme cinématographique est plus parfaite, c'est qu'elle ne connaît pas le tremblement du fait d'être au monde, du fait d' « en être ». (Zernik 104)

Une autre opposition se réfère au champ visuel de la perception : « La perception ordinaire est ouverture, tandis que la perception cinématographique est fermeture et fixation » (Zernik 105), c'est-à-dire que l'horizon de la réalité est infini, tandis que dans le cinéma, l'image perçue est encadré par l'écran. Ces aspects pourraient renvoyer aussi, comme dans le cas de Tarkovski, à une mélancolie associée à la séparation et à l'interaction, saisie surtout par la perception dans le cadre du cinéma. 
Au niveau de la chromatique et du son, les deux couleurs dominantes dans le film, le bleu froid et le jaune chaud suggèrent aussi le contraste : l'une est associée à la lumière naturelle, l'autre à la lumière artificielle, l'une est employée dans les cadres extérieurs au château, l'autre surtout dans l'intérieur. En plus, on considère que tout ce qui est perçu à distance a la tendance de prendre une teinte bleue, comme si l'atmosphère s'était densifiée, baignant les objets dans l'horizon. La grande planète bleue qui se trouve à distance est celle qui règne sur tout le film mélancolique. À propos de la bande son, qui comporte seulement le prologue à Tristan et Iseut de Richard Wagner, répété à certains moments dans le film comme un refrain, il n'est pas tout à fait en concordance avec les événements. Selon le philosophe Slavoj Žižek, la musique exprime parfois ce qui est d'une intensité émotionnelle trop grande pour être montrée à travers les images, et d'autres fois, le contraire de ce qui est visible. Dans le cas de Melancholia, si on pense à l'histoire d'amour entre Tristan et Iseut, elle est extrêmement romantique, tandis que le couple Justine-Michael ne reflète pas un tel romantisme : même s'il y a parfois des moments de tendresse, ils sont plutôt des rituels spécifiques à la fête de noces, vus dans la première partie du film, tandis que dans la seconde partie le couple est déjà séparé. De même, on sait que Tristan et Iseut sont tombés amoureux de manière très hasardeuse, après avoir bu par mégarde une potion magique. Au contraire, le mariage célébré dans le film implique un choix libre et conscient du partenaire.

Le choix d'une composition de Wagner n'est pas arbitraire, mais il soutient un autre contraste, observé dans l'évolution de la musique par le critique et musicologue André Cœuroy et rendu visible dans Melancholia, surtout dans la scène où Justine remplace les tableaux abstraits que les albums de la bibliothèque affichent avec des tableaux prémodernes appartenant au Pieter Brueghel, Everett Millais ou Le Caravage (fig. 2). Von Trier, en évitant toute influence du cinéma contemporain, envisage le retour au romantisme allemand, à « l'art intégral » et à la musique "impure », tel comme Cœuroy décrit l'œuvre de Wagner dans Wagner et l'esprit romantique. Dans son livre, le critique oppose la musique pure et l'antiwagnérisme qui caractérise l'art

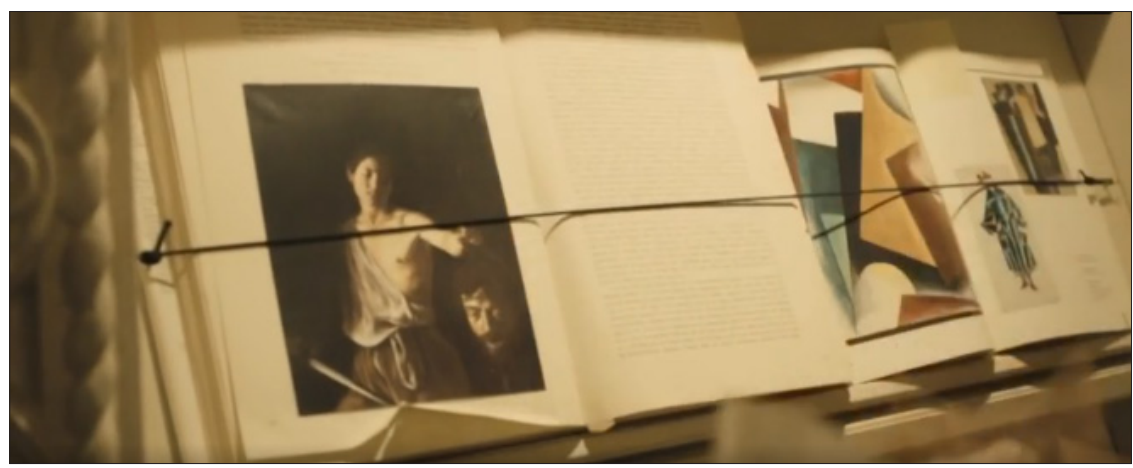

Fig. 2. Melancholia - Les catalogues d'art dans la bibliothèque. 
du début du XX $X^{\mathrm{e}}$ siècle au système et à l'idéal de l'art wagnérien. Tandis que la pureté signifie l'indépendance, c'est-à-dire que l'art ne trouve son appui et sa fin qu'en luimême, dans son propre organisme, l'impureté de Wagner implique un mélange des formes artistiques, une synthèse de poésie et musique, comme un retour au Moyen Âge et comme une continuation de l'esprit artistique allemand traditionnel. La pureté d'une peinture solide et géométrique, avec des figures et des contours bien définis, est remplacée par la fluidité d'Ophélie. Toutes les références artistiques imprègnent le film d'un état mélancolique essentiellement romantique, conforme au sentiment exprimé par la musique romantique allemande: «Quel sentiment? Le plus indéfini, le plus cosmique, le plus inexprimable, le plus littéraire. » (Cœuroy 369).

On arrive maintenant à la représentation concrète de la mélancolie dans le cinémacorps, c'est-à-dire incarnée dans le personnage de Justine et dans le film. La dépression de la jeune femme qui vient de se marier est inspirée de celle que le réalisateurmême avait vécue dès qu'il était très jeune. Il avoue la ressentir tout d'un coup, sans motivation définie, et il affirme que parfois elle ne s'apaisait que pour ressurgir encore plus intensément. Nous avons souligné ces aspects pour les reconnaître aussi chez Justine. En effet, le facteur déclencheur de son trouble mental n'est pas montré de manière évidente dans le film, on est tenté de croire que la dépression de Justine existait même avant le mariage, mais la première manifestation apparaît dans le film après un discours donné par sa mère divorcée à propos de l'inutilité de l'amour. Le " Enjoy it while it lasts » de sa mère semble anticiper le désastre qui mettra fin au mariage, mais ce n'est pas la peur d'une catastrophe qui nourrit sa condition, c'est plutôt le sentiment de la contingence et du non-sens de l'existence. La dépression ne cesse jamais, elle est seulement cachée derrière un sourire faux quand Justine se trouve à la fête, parmi les invités. Ces " pauses » sont artificielles, car, quand elle est seule, elle se laisse complètement envahie par la mélancolie, elle ne la contrôle $\mathrm{d}$ 'aucune manière, $\mathrm{d}^{\prime}$ où résulte des comportements très bizarres (fig. 3 ).

Les trois symptômes les plus évidents de Justine sont la fatigue, la perte du désir de vivre et de l'amour pour son mari. On les retrouve aux personnages principaux

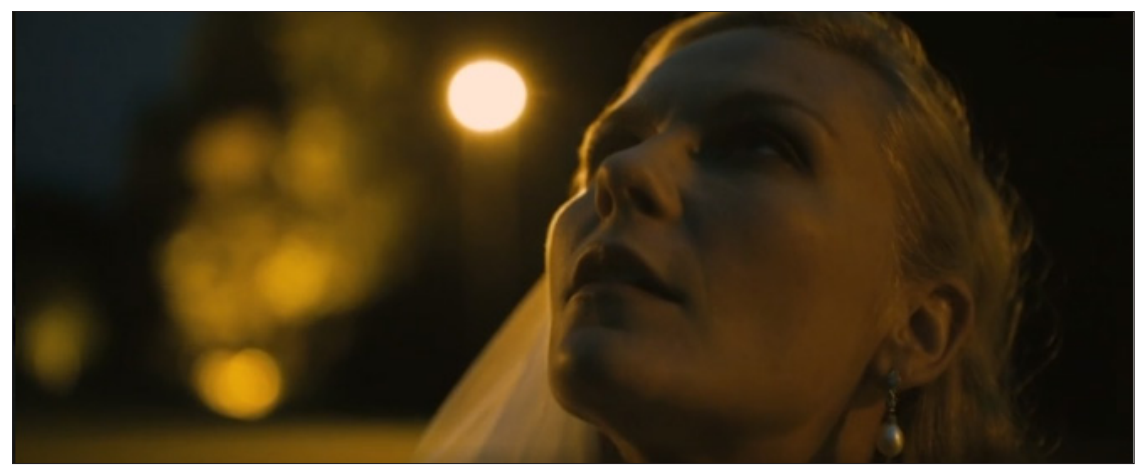

Fig. 3. Melacholia - Justine à la fête de noces. 
féminins des films d'Antonioni, dont Gilles Deleuze parle dans son Cinéma 2 : L'imagetemps, en détaillant ces troubles. On pourrait encadrer Justine dans la même typologie de femmes aliénées, qui ressentent l'angoisse existentielle, mais qui peuvent faire face mieux aux situations critiques grâce à leur pouvoir d'adaptation et à leur intuition. À la différence de sa sœur, Justine accepte la catastrophe attendue, car elle mettra fin à son angoisse, aussi qu'à l'absurdité de la vie. La femme peut pressentir le désastre, elle dit à Claire que c'est sûr que la planète va entrer en collision avec la Terre ; comment le sait-elle? Elle dit : «I just know things », tandis que les astronomes se sont trompés dans leurs calculs.

Justine sent son corps comme étant très lourd : «It's really heavy to drag along. I am trudging ", dit-elle. Son corps ressemble à ce que Deleuze nomme « le corps comme révélateur du terme » (Deleuze 247), un corps qui «n'est jamais dans le présent, il contient l'avant et l'après, la fatigue, l'attente » (Deleuze 246), qui est accablé par la pesanteur du temps, comme celui de Justine. Ce corps est très représentatif pour le processus de perception phénoménologique du corps. Selon Sartre, le corps d'autrui est toujours saisi en mouvement, comme ce qui est perpétuellement dépassé vers un but, et dont le mouvement est perçu comme allant du futur au présent (Sartre 388). Il contient aussi l'avant et l'après, c'est-à-dire un terme passé et dépassé vers le terme futur de son aboutissement. Justine ne peut pas projeter les mouvements de son corps que vers un avenir inexistant, vers la fin du monde qu'elle attend. On pourrait voir son corps non seulement comme fatigué, mais aussi comme un cadavre vivant, perçu à partir de son futur - la mort. On peut retrouver ce dépassement perpétuel dans le mouvement du film aussi, où une image et toujours en rapport avec celle qui suit et qui contribue à son sens. Ainsi résulte un film comme un corps qui révèle la mélancolie, en accord avec Antonioni, qui veut exprimer l'intérieur par le comportement, mais aussi avec Sartre, qui affirme que les manifestations émotionnelles, ou les phénomènes psychiques sont toujours comprises à travers les manifestations d'un corps.

En ce qui concerne la perte du désir d'aimer et de vivre manifestée par Justine dès son mariage, on pourrait l'interpréter du point de vue de la phénoménologie sartrienne, prenant en considération la définition de l'amour comme projet vers ses possibilités et comme appropriation d'autrui en tant que subjectivité regardante, qu'on a vu échouer dans Solaris et qui échoue aussi dans Melancholia, pas nécessairement à cause du jeu « reflet-reflété » décrit dans le cas de Solaris, mais ici surtout parce qu'il s'oppose à cette description donnée par Sartre:

[...] celui qui veut être aimé ne désire pas l'asservissement de l'être aimé. Il ne tient pas à devenir l'objet d'une passion mécanique et débordante. Il ne veut pas posséder un automatisme, et si on veut l'humilier, il suffit de lui représenter la passion de l'aimé comme le résultat d'un déterminisme psychologique : l'amant se sentira dévalorisé dans son amour et dans son être. Si Tristan et Iseut sont affolés par un philtre, ils intéressent moins ; et il 
arrive qu'un asservissement total de l'être tue l'amour de l'amant [...] Il veut posséder une liberté comme liberté. (Sartre 407)

En voyant Michael complètement asservi à elle, Justine perd son amour, aussi que sa pulsion de vie, car elle ne réussit pas à justifier sa facticité, elle continue à se sentir « de trop » dans le monde et elle risque de tomber dans des attitudes masochistes, où, dans le cas de Justine, dans le désir que la planète vienne annihiler toute l'existence.

En fait, le film en entier est une attente de la fin, état caractéristique à la mélancolie. L'attente est aussi très souvent rencontrée chez Tarkovski, surtout dans un mouvement de la caméra qui lui est spécifique : le lent glissement. La caméra glisse sur des objets ou sur des personnages dans une direction, en créant l'impression qu'à la fin de ce lent mouvement, quelque chose d'important ou d'extraordinaire nous sera révélée, mais dans la plupart de cas, cela n'arrive pas. Ce mouvement fait souvent un retour : la caméra glisse vers le point de départ initial et c'est maintenant qu'un sens se crée. Il faut faire attention à ce que la caméra nous montre pour découvrir le sens qui se cache derrière ce mouvement circulaire à titre de narration en images. En fait, cette lenteur, cette attente est ce qui crée le rythme du film, décrit par Gérard Betton comme un rythme de l'attention et par Eisenstein comme une danse (Betton 75). Comme un changement très rapide des images alimente l'attention du spectateur, la lenteur peut aussi avoir le même effet. Où est-ce que se trouve ce rythme dans Melancholia, vu que la caméra ne présente pas le même mouvement rencontré chez Tarkovski? Dans une première instance, on pourrait repérer facilement le mouvement en ralenti du début du film, qui a aussi le rôle de captiver le spectateur. Mais le véritable mouvement d'attente mélancolique se trouve dans la trajectoire de la planète, qui est même nommé dans le film «Dance of Death ». Comme chez Tarkovski, un premier déplacement n'aboutit à rien : la première fois que la planète se rapproche de la Terre, elle n'entre pas en collision. Ensuite, elle s'éloigne, en rassurant Claire, tandis que l'attente de Justine continue. Melacholia recommence son chemin et cette fois elle apporte la destruction. Ainsi, cette attente $\mathrm{du}$ « rien » prend de grandes proportions : par cette planète qui apporte la destruction, la mélancolie devient un sentiment universel, existentiel, qui est incarné dans un corps et dans un film-corps et dont le spectateur prend conscience à travers l'expérience cinématographique.

En conclusion, on a vu pourquoi il est important d'observer la mélancolie sentie dans un corps : c'est seulement de cette façon qu'elle est vraiment perçue comme vivante et comme vécue, par le mouvement des formes qui caractérise les images cinématographiques. Le recours à une approche phénoménologique de la perception nous a aidé à mieux comprendre comment elle s'exprime dans l'art cinématographique et pourquoi elle est surtout expérimentée par le cinéphile. 


\section{Notes}

1 v. Metz, "À propos de l'impression de réalité au cinéma », dans Essais sur la signification au cinéma. Tome I, Paris, Klincksieck, 1975.

2 Maurice Merleau-Ponty, "Le cinéma et la nouvelle psychologie », conférence faite le 13 mars 1945 à l'Institut des Hautes Etudes Cinématographiques et publié dans Sens et non-Sens, Paris, Les Éditions Nagel, 1966, 5e édition, collection Pensées.

3 L'amour de Kris pour Hari est semblable à notre désir irréalisable pour le cinéma. Ce que nous désirons, c'est le contact et la communication, mais paradoxalement, comme les écrans, Hari aussi fait ressortir notre solitude. Seuls, peut-être que nous serions moins conscients de notre solitude (n. t.).

4 v. Metz, Le signifiant imaginaire : psychanalyse et cinéma, Paris, Christian Bourgois, 1984.

5 Pour Jean-Paul Sartre, aimer signifie vouloir que l'autre vous aime en tant qu'objet, en gardant sa subjectivité, sans que vous le possédiez. À l'inverse, pour qu'il vous aime, il faut qu'il veut aussi la même chose, c' est-à-dire devenir objet de votre amour et soumettre volontairement sa liberté et d'ici un amour impossible (v. Sartre 404-419).

\section{Bibliographie}

Albera, François. «Maurice Merleau-Ponty et le cinéma.» 1895. Mille huit cent quatrevingt-quinze, nr. 70, 2013, pp. 120-153. Le 24 mai 2019 <http://journals.openedition. org/1895/4680>.

Betton, Gérard. Esthétique du cinéma. PUF, 1994.

Cœuroy, André. Wagner et l'esprit romantique. Gallimard, 1965.

Renegade Cut - Solaris: The Consciousness in Outer Space. Leon Thomas. 2017. Le 24 mai 2019 $<$ https://www.youtube.com/watch? $\mathrm{v}=\mathrm{c} 8 \mathrm{zcH} 2220 \mathrm{rw} \& \mathrm{t}=52 \mathrm{~s}>$.

Deleuze, Gilles. Cinéma 2 : L'image-temps. Les Éditions de Minuit, 1983.

Dillon, Steve. The Solaris Effect : Art and Artifice in Contemporary American Film. University of Texas Press, 2006.

Merleau-Ponty, Maurice. Sens et non-sens. Les Éditions Nagel, 1966.

Metz, Christian. Essais sur la signification du cinéma. Tome I. Klincksieck, 1975.

—. Le signifiant imaginaire. Psychanalyse et cinéma. Christian Bourgois, 1984.

Miller, Amy. ««Melancholia» as Wagnerian Music Drama.» Août 2015. Aristocrats of the Soul. Le 23 avril $2019<$ http://www.aristocratsofthesoul.com/melancholia-as-wagnerian-music-drama/>.

Sartre, Jean-Paul. L'être et le néant : Essai d’ontologie phénoménologique. Gallimard, 1943.

Solaris - What it means to be alive. Vaughan Supple. 2017. Le 24 mai $2019<\mathrm{https}$ //www.youtube.com/watch? $\mathrm{v}=$ an6brehsD7c\&t=31s $>$.

Zernik, Clélia. ««Un film ne se pense pas, il se perçoit» Merleau-Ponty et la perception cinématographique.» Rue Descartes, vol. 53, no. 3, 2006, pp. 102-109. Le 24 mai $2019<\mathrm{https} / /$ www.cairn.info/revue-rue-descartes-2006-3-page-102.htm>.

Cinematic Thinking. Slavoj Žižek. 2016. Le 24 mai $2019<$ https://www.youtube.com/watch? $\mathrm{v}=\mathrm{jcbv0VKSwMQ>}$. 